\title{
Association between the Time of Length since Smoking Cessation and Insulin Resistance in Asymptomatic Korean Male Ex-Smokers
}

\author{
Ko-Woon Kim, ${ }^{1}$ Sung-Goo Kang, ${ }^{1}$ Sang-Wook Song, ${ }^{1,2}$ Na-Rae Kim, ${ }^{1}$ Jun-Seung Rho, ${ }^{1}$ and \\ Yun-Ah Lee ${ }^{1}$ \\ ${ }^{1}$ Department of Family Medicine, College of Medicine, The Catholic University of Korea St. Vincent's Hospital, \\ Suwon, Republic of Korea \\ ${ }^{2}$ Health Promotion Center, College of Medicine, The Catholic University of Korea St. Vincent's Hospital, Suwon, Republic of Korea
}

Correspondence should be addressed to Sung-Goo Kang; hippo94@naver.com

Received 15 February 2017; Accepted 21 May 2017; Published 19 June 2017

Academic Editor: Samy McFarlane

Copyright @ 2017 Ko-Woon Kim et al. This is an open access article distributed under the Creative Commons Attribution License, which permits unrestricted use, distribution, and reproduction in any medium, provided the original work is properly cited.

\begin{abstract}
Aim. Smoking is a major risk factor for diabetes mellitus, mainly due to decreased insulin secretion and increased insulin resistance. However, there has been little research on the effects of smoking cessation period on changes in insulin resistance. In this study, we investigated the relationships between the length of time since smoking cessation period and insulin resistance in asymptomatic Korean male ex-smokers. Methods. A total of 851 male adults were included in this study. We considered several factors that can affect insulin resistance, and through multiple linear regression analysis, we assessed the effect the length of time since smoking cessation on insulin resistance in ex-smokers. Insulin resistance was represented as the insulin resistance index estimated by homeostasis model assessment. Results. HOMA-IR values showed a statistically significant negative correlation with the length of time since smoking cessation $(p=0.009)$ in ex-smokers. After performing multiple linear regression analysis using factors that could potentially influence insulin resistance, we found that waist circumference $(p=0.026)$ and the length of time since smoking cessation $(p=0.039)$ were independent predictors of HOMA-IR in asymptomatic male ex-smokers. Conclusion. The longer the smoking cessation period, the more the insulin resistance tended to decrease in asymptomatic Korean male ex-smokers.
\end{abstract}

\section{Introduction}

Smoking is a leading global cause of preventable death. Due to smoking, nearly 6 million people have died and hundreds of billions of dollars are spent annually worldwide to minimize smoking practices [1]. The negative effects of smoking lead to reduced quality of life and cause personal and national financial burdens.

As awareness of the importance of smoking cessation has emerged, countries around the world have implemented various smoking cessation policies. As a result of many nonsmoking policies, daily smokers, the percentage of the population aged 15 years and older declined from $19.1 \%$ in 2000 to $12.9 \%$ in 2014 in the United States of America, according to health data from the Organization from
Economic Cooperation and Development (OECD) [2]. In Korea, daily smokers decreased from $20.0 \%$ in 2014 to $17.3 \%$ in 2015 according to the OECD health data and the Korea National Health and Nutrition Examination Survey (KNHANES) [2, 3]. This suggests that smoking cessation policies such as increased in cigarette prices and the expansion of nonsmoking areas in 2015 were effective at reducing the smoking rate. However, this level has not yet reached the level of developed countries. In 2012, the rate of smokers aged 15 and over in Korea was 6th among the 34 OECD countries (21.6\%).

Smoking is a major risk factor for metabolic syndrome and cardiovascular disease [4-7]. Diabetes mellitus (DM) is one of the most common chronic diseases, with an increasing prevalence rate in the world [8], and the number of patients 
in Korea is approaching 4.8 million. It is likely that the number of individuals with insulin resistance is much greater than this estimate.

Smoking is also a major risk factor for diabetes because it decreases insulin secretion [9] and increases insulin resistance [10-12]. Many studies have shown that active smoking increases the prevalence and incidence of not only type 2 diabetes mellitus (DM) [13-15] but also glucose intolerance [16]. The causes of insulin resistance are not well defined, but both genetic and environmental factors including obesity, physical inactivity, and smoking may play a role. For example, obesity $[17,18]$ and physical inactivity $[19,20]$ are considered important risk factors for insulin resistance. Craig et al. found that smoking increases triglycerides (TG), decreases high-density lipoprotein cholesterol (HDL-C), and causes hyperinsulinemia and resistance of insulinmediated glucose uptake $[11,21,22]$.

Facchini et al. compared the prevalence of insulin resistance according to smoking status and showed that smoking was correlated with greater insulin resistance [11]. It has also been reported that smoking increases insulin resistance in a dose-dependent manner in patients with type 2 diabetes mellitus [23]. Moreover, there were almost no previous studies on changes in insulin resistance according to the length of smoking cessation period. Therefore, we investigated the relationship between the length of smoking cessation period and insulin resistance in asymptomatic Korean male exsmokers in this study.

\section{Methods}

2.1. Subjects. The initial study population included adult males who visited a university hospital in Gyeonggi province, Republic of Korea, for medical check-ups. At these checkups, information regarding the cardio-ankle vascular index (CAVI) and ankle-brachial index (ABI) were routinely assessed. The subjects were screened before they were admitted to the study. Among the 851 questionnaires, 312 people were excluded for being current-smokers and 57 subjects were excluded for not providing health-related behaviors including smoking history. Also, 77 subjects were excluded who had been diagnosed with diabetes and hypertension or were receiving treatment for such diseases or dyslipidemia; 137 subjects were also excluded who had not been tested for insulin resistance (fasting insulin and/or fasting blood sugar). Thus, 104 never-smokers and 164 ex-smokers were included in this study (Figure 1).

2.2. Ethics Statement. This study was conducted in accordance with the ethical and safety guidelines of the Institutional Review Board (IRB) at The Catholic University of Korea St. Vincent's Hospital (IRB Approval number VC17RISI0019). Because we reviewed health screening data and medical record retroactively, this study was exempted from written informed consent requirements. The IRB approved this consent procedure.

2.3. Measures. Before undergoing a physical examination, subjects provided information about their smoking status, alcohol intake, and exercise habits using self-reported questionnaires.

Smoking status was classified as never-smokers and exsmokers. Depending on what was listed in the questionnaire, alcohol consumption was defined as $N o$, drinking $<1$ time per week or Drinking, $\geq 1$ time per week; exercise was classified into Regular, $\geq 2$ time per week and $\geq 30$ minutes per time, Irregular, $<2$ time per week or $<30$ minutes per time, or No, no exercise. In this study, we measured factors that could affect insulin resistance including body mass index (BMI), waist circumference (WC), systolic blood pressure (SBP), diastolic blood pressure (DBP), TG, HDL-C, CAVI, and ABI.

Height and weight were measured with an automatic anthropometric instrument in an upright posture without shoes and socks, and BMI was calculated as body weight $(\mathrm{kg}) /$ height squared $\left(\mathrm{m}^{2}\right)$. WC was measured at the midpoint between the lower rib and the upper iliac crest. Blood pressure was measured with an automatic manometer after a stable state for more than 5 minutes.

CAVI is not influenced by blood pressure but reflects the stiffness of all blood vessels, including the aorta, the femoral artery, and the tibial artery [24]. It was measured noninvasively using a Vasera VS-1000 system. CAVI can be calculated using the following formula:

$$
\mathrm{CAVI}=a\left[\left(\frac{2 \rho}{\Delta P}\right) \times \ln \left(\frac{\mathrm{Ps}}{\mathrm{Pd}}\right) \mathrm{PWV}^{2}\right]+b
$$

(Ps is SBP, Pd is DBP, $\Delta P=\mathrm{Ps}-\mathrm{Pd}, \rho$ is blood density, PWV is pulse wave velocity, and $a$ and $b$ are constants).

After measuring blood pressure in both arms and ankles, ABI was calculated according to the American Heart Association definition by dividing the higher systolic pressure of the right and left ankle by the higher systolic pressure of both arms.

Blood samples were collected on the day of examination after at least 8 hours of fasting for analyzing factors of metabolic syndrome including fasting plasma sugar (FBS), insulin, TG, and HDL-C levels for each sample by using an automatic clinical chemistry analyzer (Hitachi 7600; Japan, and Sysmex XE-2100; Japan).

Insulin resistance indicates that the response to insulin is less than normal under a given insulin concentration. In this study, we used the homeostasis model assessment of insulin resistance (HOMA-IR) and homeostasis model assessment of beta-cell function (HOMA- $\beta$ ). HOMA-IR and HOMA- $\beta$ were calculated using the following formula:

$$
\begin{aligned}
\text { HOMA-IR } & =\frac{\text { fasting glucose }[\mathrm{mg} / \mathrm{dL}] \times \text { insulin }[\mu \mathrm{U} / \mathrm{mL}]}{405}, \\
\text { HOMA }-\beta & =\frac{20 \times \text { fasting insulin }[\mu \mathrm{U} / \mathrm{mL}]}{\text { fasting glucose }[\mathrm{mg} / \mathrm{dL}] / 18-3.5} .
\end{aligned}
$$

2.4. Statistical Analysis. The data were analyzed using the Statistical Package for Social Sciences (SPSS), version 18.0 for Windows. Using the chi-squared test, the incontinuous variables in each group were compared. The continuous variables in each of the two groups were compared using the $t$-test. Because the standard deviation of HOMA-IR and HOMA- $\beta$ 


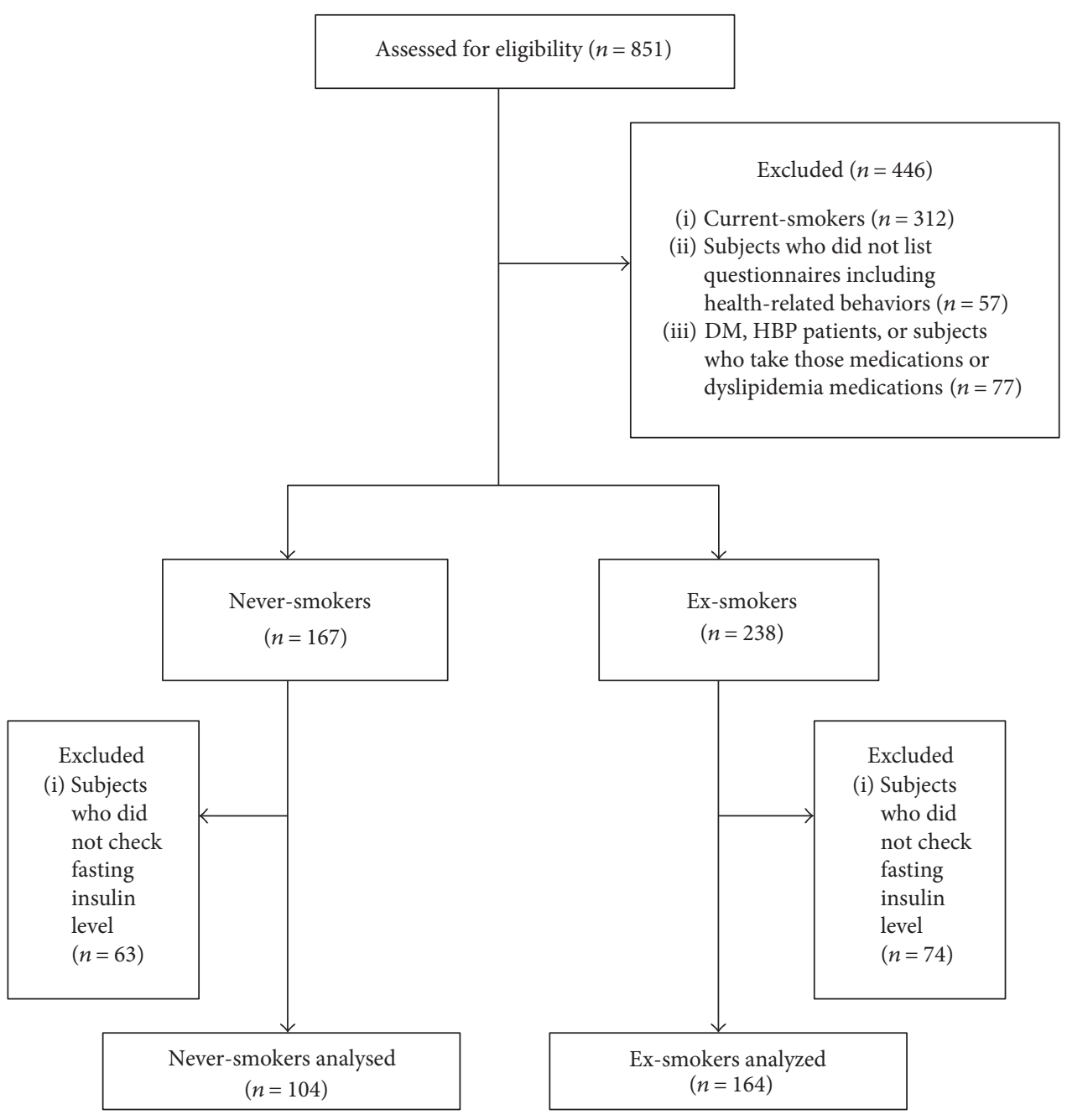

FIGURE 1: The flow chart of the study.

was large compared to the mean and not normally distributed, we used nonparametric analysis such as Mann-Whitney or Kruskal-Wallis test. We performed multivariate regression analysis to evaluate factors that affected insulin resistance. $p$ values less than 0.05 were considered statistically significant.

\section{Results}

3.1. Characteristics of Subjects. Clinical and metabolic characteristics of subjects are listed in Table 1 by smoking status. The total number of subjects was $268 ; 104$ (38.8\%) were never-smokers and $164(61.2 \%)$ were ex-smokers. No statistically significantly differences were observed between never-smokers and ex-smokers for clinical or metabolic parameters. The average duration of smoking cessation was $10.09 \pm 6.88$ years, the shortest period was 0.5 years, and the longest was 32 years.

3.2. Associations between HOMA-IR and Other Cofactors in Ex-Smokers. Although not statistically significant, the HOMA-IR values tended to decrease as the period of smoking cessation was longer when analyzed by classifying the smoking cessation period every 5 years (Table 2).
As a result of analyzing associations between HOMAIR and other cofactors in ex-smokers, HOMA-IR values showed a statistically significant positive correlation with BMI, WC, SBP, DBP, and TG. Moreover, HOMA-IR values showed a statistically significant negative correlation with the time elapsed since smoking cessation $(p=0.009)$ and HDL-C $(p=0.003)$. No statistically significant correlation was found between HOMA-IR values and age, CAVI, or ABI (Table 3).

3.3. Factors That Affect Insulin Resistance in Ex-Smokers. After performing multiple linear regression analyses, with factors that can potentially influence insulin resistance, we found that WC and the time elapsed since smoking cessation were independent predictors of HOMA-IR in asymptomatic Korean male ex-smokers (Table 4). However, the duration of the smoking cessation period and HOMA- $\beta$ values were not significantly correlated.

\section{Discussion}

Many studies have shown that smoking is associated with insulin resistance [10-12]. However, studies on the 
TABLE 1: Baseline characteristics of the study population according to smoking status.

\begin{tabular}{|c|c|c|c|}
\hline & $\begin{array}{l}\text { Never-smokers } \\
\quad(n=104)\end{array}$ & $\begin{array}{c}\text { Ex-smokers } \\
(n=164)\end{array}$ & $p$ value \\
\hline Age (years) & $54.09 \pm 8.07$ & $54.34 \pm 7.50$ & 0.797 \\
\hline BMI $\left(\mathrm{kg} / \mathrm{m}^{2}\right)$ & $24.30 \pm 2.69$ & $24.58 \pm 2.81$ & 0.420 \\
\hline $\mathrm{WC}(\mathrm{cm})$ & $86.42 \pm 6.39$ & $86.96 \pm 6.53$ & 0.500 \\
\hline SBP (mmHg) & $128.84 \pm 14.02$ & $129.93 \pm 14.29$ & 0.540 \\
\hline DBP (mmHg) & $77.24 \pm 10.05$ & $78.84 \pm 9.47$ & 0.191 \\
\hline Alcohol use & & & 0.181 \\
\hline No & $39(44.8 \%)$ & $48(55.2 \%)$ & \\
\hline Yes $^{\mathrm{a}}$ & $65(35.9 \%)$ & $116(64.1 \%)$ & \\
\hline Exercise & & & 0.383 \\
\hline Regular $^{\mathrm{b}}$ & $38(35.5 \%)$ & $69(64.5 \%)$ & \\
\hline Irregular $^{c}$ & $37(37.8 \%)$ & $61(62.2 \%)$ & \\
\hline $\mathrm{No}^{\mathrm{d}}$ & $29(46.0 \%)$ & $34(54.0 \%)$ & \\
\hline HDL-C (mg/dL) & $45.00 \pm 10.87$ & $45.79 \pm 9.25$ & 0.524 \\
\hline $\mathrm{TG}(\mathrm{mg} / \mathrm{dL})$ & $130.92 \pm 80.16$ & $145.11 \pm 97.46$ & 0.215 \\
\hline $\mathrm{FBS}(\mathrm{mg} / \mathrm{dL})$ & $95.36 \pm 14.33$ & $97.13 \pm 13.91$ & 0.314 \\
\hline INSULIN $(\mu \mathrm{U} / \mathrm{mL})$ & $3.73 \pm 4.57$ & $4.03 \pm 3.76$ & 0.547 \\
\hline HOMA-IR & $0.89 \pm 1.07$ & $0.98 \pm 0.96$ & 0.333 \\
\hline HOMA- $\beta$ & $44.32 \pm 55.27$ & $46.02 \pm 43.23$ & 0.643 \\
\hline CAVI & $7.61 \pm 0.99$ & $7.50 \pm 0.84$ & 0.324 \\
\hline $\mathrm{ABI}$ & $1.15 \pm 0.07$ & $1.15 \pm 0.07$ & 0.650 \\
\hline
\end{tabular}

$p$ value was calculated by $t$-test or Mann-Whitney test for continuous variables or chi-square test for incontinuous variables. Values are presented as mean \pm SD or $\mathrm{n}(\%)$. ${ }^{\mathrm{a}}$ Drinking $\geq 1$ time per week. ${ }^{\mathrm{b}}$ Exercise $\geq 1$ time per week and $\geq 30$ minutes per time. ${ }^{\mathrm{c}}$ Exercise $<1$ time per week or $<30$ minutes per time. ${ }^{\mathrm{d}} \mathrm{No}$ exercises. BMI: body mass index; WC: waist circumference; SBP: systolic blood pressure; DBP: diastolic blood pressure; FBS: fasting blood sugar; TG: triglycerides; HDL-C: high-density lipoprotein cholesterol; HOMA-IR: homeostasis model assessment of insulin resistance; CAVI: cardio-ankle vascular index; ABI: ankle-brachial index.

TABLE 2: HOMA-IR values according to 5 years smoking cessation categories in ex-smokers.

\begin{tabular}{llll}
\hline Nonsmoking period (years) & $N$ & Mean \pm SD & $p$ value \\
\hline $0-5$ & 52 & $1.28 \pm 1.28$ & \\
$6-10$ & 55 & $0.93 \pm 0.87$ & \\
$11-15$ & 29 & $0.80 \pm 0.61$ & 0.118 \\
$\geq 16$ & 28 & $0.98 \pm 0.96$ & \\
\hline
\end{tabular}

$p$ value was obtained by Kruskal-Wallis analysis.

relationship between smoking cessation period and insulin resistance are rare. Given these findings, we assessed the association between the length of time since smoking cessation and HOMA-IR values (as an indicator of insulin resistance) in asymptomatic Korean male ex-smokers.

We found that HOMA-IR values had a statistically significant negative correlation with the duration of the period since smoking cessation in ex-smokers. After performing multiple linear regression analysis, only WC and the duration of time since smoking cessation showed a statistically negative correlation with HOMA-IR values in ex-smokers. According to the Korea Diabetes Status Report in 2016, the prevalence of impaired fasting glucose (IFG) in Korea is
TABLE 3: The correlation coefficient $(r)$ between HOMA-IR and other cofactors in ex-smokers.

\begin{tabular}{lcc}
\hline & $r$ & $p$ value \\
\hline Age $($ years $)$ & -.038 & 0.625 \\
BMI $\left(\mathrm{kg} / \mathrm{m}^{2}\right)$ & .456 & $<0.001$ \\
WC $(\mathrm{cm})$ & .484 & $<0.001$ \\
Nonsmoking period & -.203 & 0.009 \\
SBP $(\mathrm{mmHg})$ & .220 & 0.005 \\
DBP $(\mathrm{mmHg})$ & .280 & $<0.001$ \\
HDL-C $(\mathrm{mg} / \mathrm{dL})$ & -.233 & 0.003 \\
TG $(\mathrm{mg} / \mathrm{dL})$ & .350 & $<0.001$ \\
CAVI & -.056 & 0.479 \\
ABI & .057 & 0.470 \\
\hline
\end{tabular}

$p$ value was calculated by Pearson correlation. BMI: body mass index; WC: waist circumference; SBP: systolic blood pressure; DBP: diastolic blood pressure; HDL-C: high-density lipoprotein cholesterol; TG: triglycerides; CAVI: cardio-ankle vascular index; ABI: ankle-brachial index.

$24.8 \%$, which means that one in every four people has prediabetes [25]. And, it is well known that smoking is a risk factor of DM. From this perspective, the results of this study are meaningful that long periods of smoking cessation might contribute to lowering the prevalence of diabetes, which 
TABLE 4: Multiple linear regression between HOMA-IR and other cofactors in ex-smokers.

\begin{tabular}{lcc}
\hline Variables & $\beta$ & $p$ value \\
\hline Age $($ years $)$ & -.004 & 0.687 \\
BMI $\left(\mathrm{kg} / \mathrm{m}^{2}\right)$ & .037 & 0.451 \\
WC $(\mathrm{cm})$ & .048 & 0.026 \\
Alcohol & .275 & 0.076 \\
Exercise & .154 & 0.091 \\
Nonsmoking period & -.020 & 0.039 \\
SBP $(\mathrm{mmHg})$ & .002 & 0.812 \\
DBP $(\mathrm{mmHg})$ & .007 & 0.560 \\
HDL-C $(\mathrm{mg} / \mathrm{dL})$ & -.005 & 0.528 \\
TG $(\mathrm{mg} / \mathrm{dL})$ & .001 & 0.397 \\
CAVI & -.034 & 0.664 \\
ABI & .517 & 0.562 \\
\hline
\end{tabular}

BMI: body mass index; WC: waist circumference; SBP: systolic blood pressure; DBP: diastolic blood pressure; HDL-C: high-density lipoprotein cholesterol; TG: triglycerides; CAVI: cardio-ankle vascular index; ABI: ankle-brachial index.

might also be another basis for implementing smoking cessation policies.

In addition to these findings that the length of time since smoking cessation in ex-smokers was negatively correlated with insulin resistance, this study confirmed that WC is the most reliable predictor of insulin resistance. The findings of the present study are in line with many other studies. In fact, many studies found that current smokers are more likely to have higher WC [26-28] values than nonsmokers. However, Berlin et al. found that high WC values were less frequent among current smokers [29]. Similarly, some studies have assessed the relationship between smoking and waist-hip ratio (WHR); Visser et al. found that current-smokers had the highest WHR and never-smokers the lowest [30]. Barrett-Connor and Khaw found that smokers had a higher WHR than never-smokers and observed a dose-response relationship between increasing WHR and increasing number of cigarettes smoked in both sexes [31]. Further research is needed on the mechanism by which cigarette smoking is correlated with insulin resistance.

In our study, the HOMA-IR value decreased by 0.02 annually after quitting smoking, and it also decreased by 0.048 when waist circumference decreased by $1 \mathrm{~cm}$. In other words, we could estimate that the decrease of HOMA-IR value at 10 -year smoking cessation was almost similar to the effect of $4 \mathrm{~cm}$ decrease in waist circumference. In this respect, the effect of the length of time since smoking cessation on insulin resistance might be important in that decreased waist circumference is the most reliable indicator of insulin resistance reduction.

Early vascular alterations, such as increased arterial stiffness and vascular endothelial cell injury, might be responsible for the development of the metabolic syndrome [32, 33]. Smoking may indeed accelerate those pathways. Actually, increased arterial stiffness was seen in smokers with diabetes mellitus [34], hypertension [35], and dyslipidemia [36, 37]. In our study, on the contrary, CAVI and ABI did not show a statistically significant negative correlation with HOMAIR in ex-smokers. These findings are contrary to baseline characteristic data about CAVI and ABI of ex-smokers and never-smokers, which suggests that CAVI and ABI might not be sensitive enough to measure the recovery of vascular damage during smoking cessation.

This study has a few limitations. First, it may be difficult to generalize the results of this study because the study population was not large and, because all subjects voluntarily visited the health promotion center, there might have been a selection bias. Also, because the participants in this study were Korean, these results might not directly apply people of other races. In fact, there was a study about that high nicotine concentration was associated with compromised insulin secretion among non-Hispanic Whites and nonHispanic Blacks, but not among Mexican Americans and other Hispanics [38].

Second, women were excluded from this study. In Korean culture, women's smoking tends to be concealed and the reliability of women's smoking statistics from surveys may be poor. In fact, in the KNHANES (2008), the self-reported female smoking rate was $5.9 \%$, but the smoking rate was 2.36 times higher according to urine cotinine (13.9\%) [39]. Third, we did not consider smoking amount and duration of smoking in ex-smokers before smoking cessation.

Despite these potential limitations, the present findings support the conclusion that the length of time since smoking cessation could be an independent predictor of insulin resistance in asymptomatic Korean male ex-smokers. This finding implies that smoking cessation can delay the onset of $\mathrm{DM}$, which indicates the importance of smoking cessation in the primary prevention of DM.

\section{Conflicts of Interest}

There is no conflict of interests regarding the publication of this paper.

\section{Authors' Contributions}

Sung-Goo Kang conceived and designed the experiments. Jun-Seung Rho and Na-Rae Kim performed the experiments. Sung-Goo Kang and Ko-Woon Kim analyzed the data. Sang-Wook Song and Yun-Ah Lee contributed reagents/ materials/analysis tools. Sung-Goo Kang and Ko-Woon Kim wrote the paper.

\section{References}

[1] Organization WH, WHO Report on the Global Tobacco Epidemic, 2011: Warning about the Dangers of Tobacco, World Health Organization, 2011, http://www.who.int/tobacco/ global_report/2011/exec_summary/en/.

[2] OECD, https://data.oecd.org/healthrisk/daily-smokers.htm.

[3] Korea National Health and Nutrition Examination Survey, 2015, https://knhanes.cdc.go.kr/knhanes/index.do.

[4] The pooling project research group, "Relationship of blood pressure, serum cholesterol, smoking habit, relative weight and ECG abnormalities to incidence of major coronary event: 
final report of the pooling project," Journal of Chronic Diseases, vol. 31, no. 4, pp. 201-306, 1978.

[5] R. Shinton and G. Beevers, "Meta-analysis of relation between cigarette smoking and stroke," BMJ (Clinical Research Ed), vol. 298, no. 6676, pp. 789-794, 1989.

[6] W. B. Kannel, "Update on the role of cigarette smoking in coronary artery disease," American Heart Journal, vol. 101, no. 3, pp. 319-328, 1981.

[7] K. Sun, J. Liu, and G. Ning, "Active smoking and risk of metabolic syndrome: a meta-analysis of prospective studies," PLoS One, vol. 7, no. 10, article e47791, 2012.

[8] J. E. Shaw, R. A. Sicree, and P. Z. Zimmet, "Global estimates of the prevalence of diabetes for 2010 and 2030," Diabetes Research and Clinical Practice, vol. 87, no. 1, pp. 4-14, 2010.

[9] A. Morimoto, Y. Tatsumi, K. Deura, S. Mizuno, Y. Ohno, and S. Watanabe, "Impact of cigarette smoking on impaired insulin secretion and insulin resistance in Japanese men: the Saku study," Journal of Diabetes Investigation, vol. 4, no. 3, pp. 274-280, 2013.

[10] T. Ronnemaa, E. M. Ronnemaa, P. Puukka, K. Pyorala, and L. Laakso, "Smoking is independently associated with high plasma insulin levels in nondiabetic men," Diabetes Care, vol. 19, no. 11, pp. 1229-1232, 1996.

[11] F. S. Facchini, C. B. Hollenbeck, J. Jeppesen, Y. D. Chen, and G. M. Reaven, "Insulin resistance and cigarette smoking," Lancet (London, England), vol. 339, no. 8802, pp. 1128-1130, 1992.

[12] A. Chiolero, D. Faeh, F. Paccaud, and J. Cornuz, "Consequences of smoking for body weight, body fat distribution, and insulin resistance," The American Journal of Clinical Nutrition, vol. 87, no. 4, pp. 801-809, 2008.

[13] I. Berlin, "Smoking-induced metabolic disorders: a review," Diabetes \& Metabolism, vol. 34, no. 4, Part 1, pp. 307-314, 2008.

[14] C. Willi, P. Bodenmann, W. A. Ghali, P. D. Faris, and J. Cornuz, "Active smoking and the risk of type 2 diabetes: a systematic review and meta-analysis," Jama, vol. 298, no. 22, pp. 2654-2664, 2007.

[15] H. C. Yeh, B. B. Duncan, M. I. Schmidt, N. Y. Wang, and F. L. Brancati, "Smoking, smoking cessation, and risk for type 2 diabetes mellitus: a cohort study," Annals of Internal Medicine, vol. 152, no. 1, pp. 10-17, 2010.

[16] T. K. Houston, S. D. Person, M. J. Pletcher, K. Liu, C. Iribarren, and C. I. Kiefe, "Active and passive smoking and development of glucose intolerance among young adults in a prospective cohort: CARDIA study," BMJ (Clinical Research Ed), vol. 332, no. 7549, pp. 1064-1069, 2006.

[17] B. B. Kahn and J. S. Flier, "Obesity and insulin resistance," The Journal of Clinical Investigation, vol. 106, no. 4, pp. 473-478, 2000.

[18] E. J. Gallagher, D. Leroith, and E. Karnieli, "Insulin resistance in obesity as the underlying cause for the metabolic syndrome," The Mount Sinai Journal of Medicine, New York, vol. 77, no. 5, pp. 511-523, 2010.

[19] D. J. Magliano, E. L. Barr, P. Z. Zimmet et al., "Glucose indices, health behaviors, and incidence of diabetes in Australia: the Australian Diabetes, Obesity and Lifestyle study," Diabetes Care, vol. 31, no. 2, pp. 267-272, 2008.

[20] D. Mozaffarian, A. Kamineni, M. Carnethon, L. Djousse, K. J. Mukamal, and D. Siscovick, "Lifestyle risk factors and newonset diabetes mellitus in older adults: the cardiovascular health study," Archives of Internal Medicine, vol. 169, no. 8, pp. 798-807, 2009.
[21] W. Y. Craig, G. E. Palomaki, and J. E. Haddow, "Cigarette smoking and serum lipid and lipoprotein concentrations: an analysis of published data," BMJ (Clinical Research Ed), vol. 298, no. 6676, pp. 784-788, 1989.

[22] W. Willett, C. H. Hennekens, W. Castelli et al., "Effects of cigarette smoking on fasting triglyceride, total cholesterol, and HDL-cholesterol in women," American Heart Journal, vol. 105, no. 3, pp. 417-421, 1983.

[23] T. Ohkuma, M. Iwase, H. Fujii et al., "Dose- and timedependent association of smoking and its cessation with glycemic control and insulin resistance in male patients with type 2 diabetes mellitus: the Fukuoka Diabetes Registry," PLoS One, vol. 10, no. 3, article e0122023, 2015.

[24] K. Shirai, J. Utino, K. Otsuka, and M. Takata, “A novel blood pressure-independent arterial wall stiffness parameter; cardioankle vascular index (CAVI)," Journal of Atherosclerosis and Thrombosis, vol. 13, no. 2, pp. 101-107, 2006.

[25] KOREAN DIABETES ASSOCIATION, Korea Centers for Disease Control and Prevention, Diabetes fact sheet in Korea, 2016, http://www.diabetes.or.kr/pro/news/index.php?code= board\&number $=1332 \&$ mode $=$ view.

[26] H. Shimokata, D. C. Muller, and R. Andres, "Studies in the distribution of body fat. III. Effects of cigarette smoking," Jama, vol. 261, no. 8, pp. 1169-1173, 1989.

[27] A. Onat, H. Özhan, A. M. Esen et al., "Prospective epidemiologic evidence of a "protective" effect of smoking on metabolic syndrome and diabetes among Turkish women-without associated overall health benefit," Atherosclerosis, vol. 193, no. 2, pp. 380-388, 2007.

[28] D. Albanes, D. Y. Jones, M. S. Micozzi, and M. E. Mattson, "Associations between smoking and body weight in the US population: analysis of NHANES II," American Journal of Public Health, vol. 77, no. 4, pp. 439-444, 1987.

[29] I. Berlin, S. Lin, J. A. Lima, and A. G. Bertoni, "Smoking status and metabolic syndrome in the multi-ethnic study of atherosclerosis. A cross-sectional study," Tobacco Induced Diseases, vol. 10, no. 1, p. 9, 2012.

[30] M. Visser, L. J. Launer, P. Deurenberg, and D. J. Deeg, "Past and current smoking in relation to body fat distribution in older men and women," The Journals of Gerontology Series a Biological Sciences and Medical Sciences, vol. 54, no. 6, pp. M293-M298, 1999.

[31] E. Barrett-Connor and K. T. Khaw, "Cigarette smoking and increased central adiposity," Annals of Internal Medicine, vol. 111, no. 10, pp. 783-787, 1989.

[32] H. Yokoyama, M. Kuramitsu, S. Kanno, J. Tada, Y. Yokota, and F. Kamikawa, "Relationship between metabolic syndrome components and vascular properties in Japanese type 2 diabetic patients without cardiovascular disease or nephropathy," Diabetes Research and Clinical Practice, vol. 75, no. 2, pp. 200-206, 2007.

[33] H. O. Steinberg, H. Chaker, R. Leaming, A. Johnson, G. Brechtel, and A. D. Baron, "Obesity/insulin resistance is associated with endothelial dysfunction. Implications for the syndrome of insulin resistance," The Journal of Clinical Investigation, vol. 97, no. 11, pp. 2601-2610, 1996.

[34] M. Koizumi, H. Shimizu, K. Shimomura et al., "Relationship between hyperinsulinemia and pulse wave velocity in moderately hyperglycemic patients," Diabetes Research and Clinical Practice, vol. 62, no. 1, pp. 17-21, 2003.

[35] M. F. O'Rourke, J. A. Staessen, C. Vlachopoulos, D. Duprez, and G. E. Plante, "Clinical applications of arterial stiffness; 
definitions and reference values," American Journal of Hypertension, vol. 15, no. 5, pp. 426-444, 2002.

[36] E. ter Avest, S. Holewijn, S. J. Bredie, L. J. van Tits, A. F. Stalenhoef, and J. de Graaf, "Pulse wave velocity in familial combined hyperlipidemia," American Journal of Hypertension, vol. 20, no. 3, pp. 263-269, 2007.

[37] A. Laws and G. M. Reaven, "Evidence for an independent relationship between insulin resistance and fasting plasma HDL-cholesterol, triglyceride and insulin concentrations," Journal of Internal Medicine, vol. 231, no. 1, pp. 25-30, 1992.

[38] R. Liu, Z. Zheng, J. Du, K. K. Christoffel, and X. Liu, "Racial disparity in the associations of cotinine with insulin secretion: data from the National Health and Nutrition Examination Survey, 2007-2012," PLoS One, vol. 11, no. 12, article e0167260, 2016.

[39] K. H. Jung-Choi, Y. H. Khang, and H. J. Cho, "Hidden female smokers in Asia: a comparison of self-reported with cotinineverified smoking prevalence rates in representative national data from an Asian population," Tobacco Control, vol. 21, no. 6, pp. 536-542, 2012. 


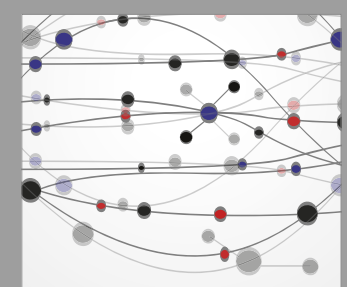

The Scientific World Journal
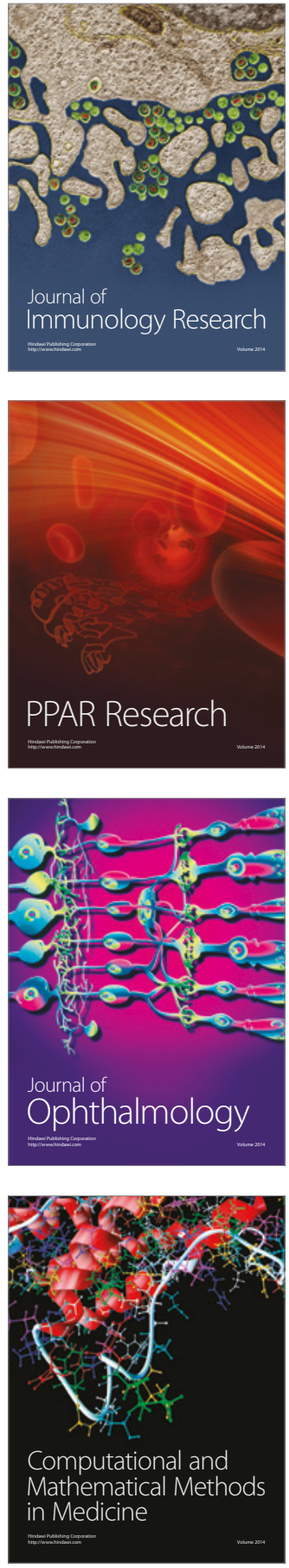

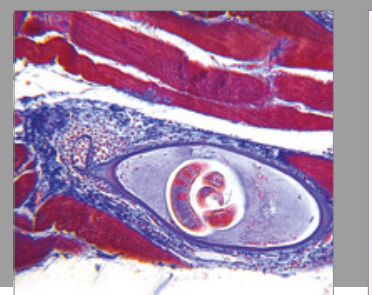

Gastroenterology Research and Practice
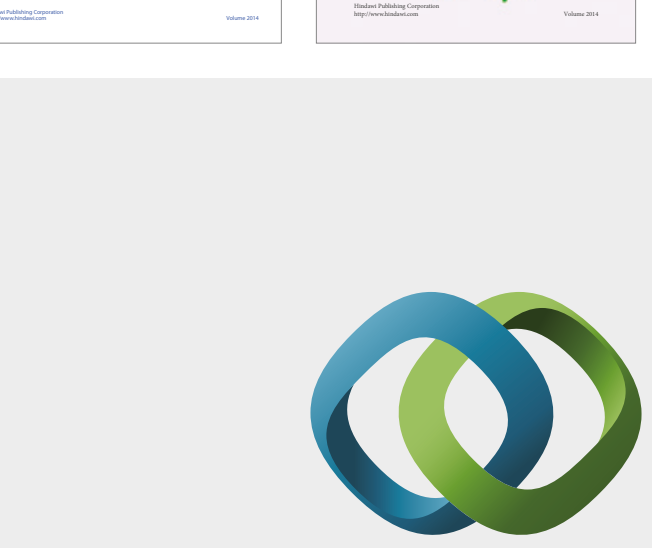

\section{Hindawi}

Submit your manuscripts at

https://www.hindawi.com
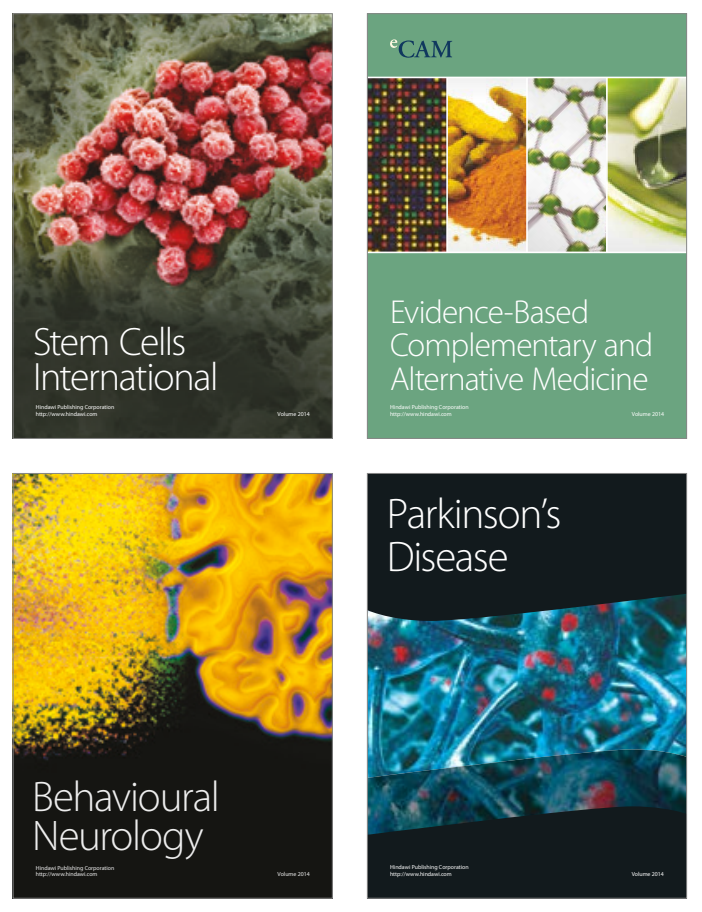
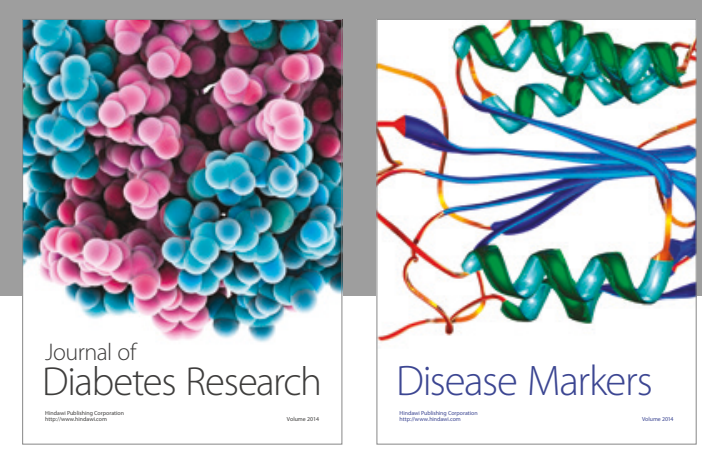

Disease Markers
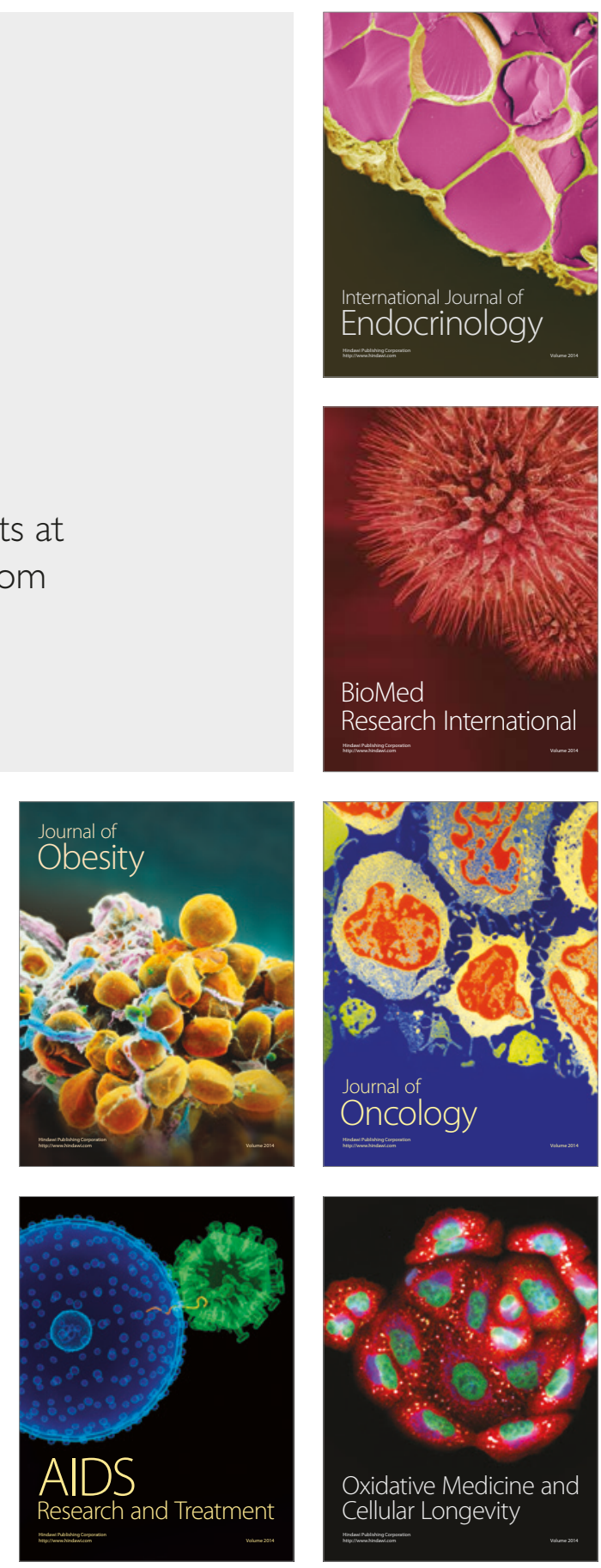